\title{
Overview on iXblue Photonics and focus on ModBox-FrontEnd Solution
}

\author{
Hervé Gouraud \\ iXblue Photonics - 3, rue Sophie Germain, F-25000 Besançon, France
}

iXblue is a global high-tech company specializing in the design and manufacturing of advanced autonomous, marine and photonics technologies. iXblue Photonics is a division of iXblue, it helps photonics engineers all around the world to get the most out of the light by providing high performance, innovative and reliable photonic solutions. iXblue Photonics solutions are dedicated to diverse industrial applications such as Telecom, Sensing, Defense, Space, Fiber Laser, Harsh Environment.

The Modulation Solutions Division from iXblue Photonics is specialized in the design and manufacture from low to $70 \mathrm{GHz}$ high frequency $\mathrm{LiNbO}_{3}$ of optical fibered modulators, and matching components such as microwave amplifiers and electronics.

The Specialty Fibers Division from iXblue Photonics develops and manufactures a wide range of specialty optical fibers, fiber optic components and subassemblies. iXblue specialty fibers and components are produced in environmentally controlled production areas to ensure high proof strength, tight tolerances and a high-quality level.

By mastering all the building blocks of an efficient modulation system, iXblue is capable to offer complex optoelectronic modulation solutions based on proprietary designs such as the ModBoxFrontEnd. The ModBox-FrontEnd is an optical sub-ns pulse shaping laser: it is a carefully tuned turnkey solution dedicated to a specific High Energy or High-Power class laser. The ModBox-FrontEnd is used to seed high power amplifier chains to produce a master laser or an OPCPA pump laser. Applications range from scientific research and academic activities to industrial market needs. The ModBoxFrontEnd operates in many different fields such as particle acceleration (GSI), inertial confinement fusion (LLNL), radiation therapy, X-ray generation, for sensor test applications (Arété Associates), laser-driven shock compression for laser peening (Hilase) or dynamic compression (ESRF). iXblue offers dedicated industrial grade Fibre Front End Seed Sources for all industries including R\&D, Defense or production.

"The ability to shape the pulse temporally is of critical importance": the ModBox-FrontEnd will challenge the state of the art of temporal pulse shaping performance:

- Operating wavelength $1030 \mathrm{~nm}, 1053 \mathrm{~nm}, 1064 \mathrm{~nm}$

- $\quad$ Output pulse energy $1 \mathrm{~nJ}$ per ns

- Very high pulse contrast up to $60 \mathrm{~dB}$

- Excellent output power stability (typically $< \pm 1 \%$ peak-to-peak)

- 125 ps - 100 ns tunable pulse duration with pulse shaping capability

- $\quad$ Up to $100 \mathrm{kHz}$ repetition rate

- $\quad$ Reliable $24 / 7$ operation

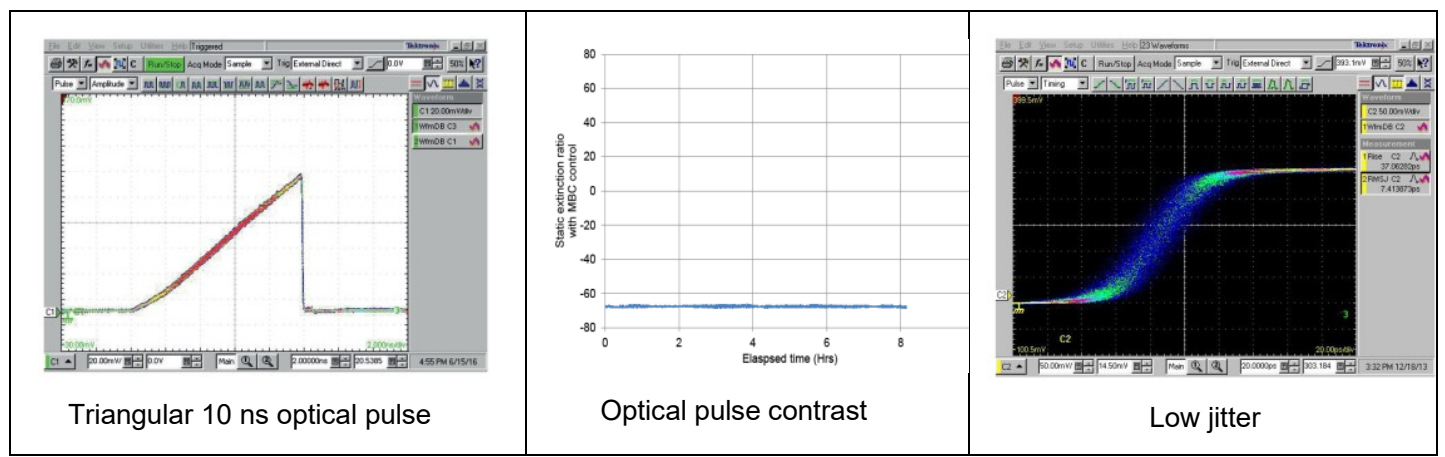

Fig. 1: Pulse Shaping and ModBox optical performances 